\title{
On the Existence of Positive Periodic Solutions for Second-Order Functional Differential Equations with Multiple Delays
}

\author{
Qiang Li and Yongxiang Li \\ Department of Mathematics, Northwest Normal University, Lanzhou 730070, China \\ Correspondence should be addressed to Yongxiang Li, liyxnwnu@163.com
}

Received 30 August 2012; Accepted 1 November 2012

Academic Editor: K. Sadarangani

Copyright (C) 2012 Q. Li and Y. Li. This is an open access article distributed under the Creative Commons Attribution License, which permits unrestricted use, distribution, and reproduction in any medium, provided the original work is properly cited.

The existence results of positive $\omega$-periodic solutions are obtained for the second-order functional differential equation with multiple delays $u^{\prime \prime}(t)+a(t) u(t)=f\left(t, u(t), u\left(t-\tau_{1}(t)\right), \ldots, u\left(t-\tau_{n}(t)\right)\right)$, where $a(t) \in C(\mathbb{R})$ is a positive $\omega$-periodic function, $f: \mathbb{R} \times[0,+\infty)^{n+1} \rightarrow[0,+\infty)$ is a continuous function which is $\omega$-periodic in $t$, and $\tau_{1}(t), \ldots, \tau_{n}(t) \in C(\mathbb{R},[0,+\infty))$ are $\omega$-periodic functions. The existence conditions concern the first eigenvalue of the associated linear periodic boundary problem. Our discussion is based on the fixed-point index theory in cones.

\section{Introduction}

In this paper, we deal with the existence of positive periodic solution of the second-order functional differential equation with multiple delays

$$
u^{\prime \prime}(t)+a(t) u(t)=f\left(t, u(t), u\left(t-\tau_{1}(t)\right), \ldots, u\left(t-\tau_{n}(t)\right)\right), \quad t \in \mathbb{R},
$$

where $a(t) \in C(\mathbb{R})$ is a positive $\omega$-periodic function, $f: \mathbb{R} \times[0,+\infty)^{n+1} \rightarrow[0,+\infty)$ is a continuous function which is $\omega$-periodic in $t$, and $\tau_{1}(t), \ldots, \tau_{n}(t) \in C(\mathbb{R},[0,+\infty))$ are $\omega$-periodic functions $\omega>0$ is a constant.

In recent years, the existence of periodic solutions for second-order functional differential equations has been researched by many authors see [1-8] and references therein. In some practice models, only positive periodic solutions are significant. In [4-8], the authors obtained the existence of positive periodic solutions for some second-order functional 
differential equations by using fixed-point theorems of cone mapping. Especially in [5], Wu considered the second-order functional differential equation

$$
u^{\prime \prime}(t)+a(t) u(t)=\lambda f\left(t, u\left(t-\tau_{1}(t)\right), \ldots, u\left(t-\tau_{n}(t)\right)\right), \quad t \in \mathbb{R},
$$

and obtained the existence results of positive periodic solutions by using the Krasnoselskii fixed-point theorem of cone mapping when the coefficient $a(t)$ satisfies the condition that $0<a(t)<\pi^{2} / \omega^{2}$ for every $t \in \mathbb{R}$. And in [8], Li obtained the existence results of positive $\omega$-periodic solutions for the second-order differential equation with constant delays

$$
-u^{\prime \prime}(t)+a(t) u(t)=f\left(t, u\left(t-\tau_{1}\right), \ldots, u\left(t-\tau_{n}\right)\right), \quad t \in \mathbb{R},
$$

by employing the fixed-point index theory in cones. For the second-order differential equations without delay, the existence of positive periodic solutions has been discussed by more authors, see [9-14].

Motivated by the paper mentioned above, we research the existence of positive periodic solutions of (1.1). We aim to obtain the essential conditions on the existence of positive periodic solution of (1.1) by constructing a special cone and applying the fixed-point index theory in cones.

In this paper, we assume the following conditions:

(H1) $a \in C(\mathbb{R},(0,+\infty))$ is $\omega$-periodic function and there exists a constant $1 \leq p \leq+\infty$ such that

$$
\|a\|_{p} \leq K\left(2 p^{*}\right)
$$

where $\|a\|_{p}$ is the $p$-norm of $a$ in $L^{p}[0, \omega], p^{*}$ is the conjugate exponent of $p$ defined by $(1 / p)+\left(1 / p^{*}\right)=1$, and the function $K(q)$ is defined by

$$
K(q)= \begin{cases}\frac{2 \pi}{q \omega^{1+2 / q}}\left(\frac{2}{2+q}\right)^{1-2 / q}\left(\frac{\Gamma(1 / q)}{\Gamma(1 / 2+1 / q)}\right)^{2}, & \text { if } 1 \leq q<+\infty \\ \frac{4}{\omega}, & \text { if } q=+\infty\end{cases}
$$

in which $\Gamma$ is the Gamma function.

(H2) $f \in C\left(\mathbb{R} \times[0,+\infty)^{n+1},[0,+\infty)\right)$ and $f\left(t, x_{0}, x_{1}, \ldots, x_{n}\right)$ is $\omega$-periodic in $t$.

(H3) $\tau_{1}(t), \ldots, \tau_{n}(t) \in C(\mathbb{R},[0, \infty))$ are $\omega$-periodic functions. the condition

In Assumption (H1), if $p=+\infty$, since $K(2)=\pi^{2} / \omega^{2}$, then (1.4) implies that $a$ satisfies

$$
0<a(t) \leq \frac{\pi^{2}}{\omega^{2}}, \quad t \in[0, \omega]
$$

This condition includes the case discussed in [5]. 
The techniques used in this paper are completely different from those in [5]. Our results are more general than those in [5] in two aspects. Firstly, we relax the conditions of the coefficient $a(t)$ appeared in an equation in [5] and expand the range of its values. Secondly, by constructing a special cone and applying the fixed-point index theory in cones, we obtain the essential conditions on the existence of positive periodic solutions of (1.1). The conditions concern the first eigenvalue of the associated linear periodic boundary problem, which improve and optimize the results in [5]. To our knowledge, there are very few works on the existence of positive periodic solutions for the above functional differential equations under the conditions concerning the first eigenvalue of the corresponding linear equation.

Our main results are presented and proved in Section 3. Some preliminaries to discuss (1.1) are presented in Section 2.

\section{Preliminaries}

In order to discuss (1.1), we consider the existence of $\omega$-periodic solution of the corresponding linear differential equation

$$
u^{\prime \prime}+a(t) u=h(t), \quad t \in \mathbb{R}
$$

where $h \in C(\mathbb{R})$ is a $\omega$-periodic function. It is obvious that finding an $\omega$-periodic solution of (2.1) is equivalent to finding a solution of the linear periodic boundary value problem

$$
\begin{aligned}
& u^{\prime \prime}+a(t) u=h(t), \quad t \in[0, \omega], \\
& u(0)=u(\omega), \quad u^{\prime}(0)=u^{\prime}(\omega) .
\end{aligned}
$$

In [14], Torres show the following existence resulted.

Lemma 2.1. Assume that (H1) holds, then for every $h \in C[0, \omega]$, the linear periodic boundary problem (2.2) has a unique solution expressed by

$$
u(t)=\int_{0}^{\omega} G(t, s) h(s) d s, \quad t \in[0, \omega]
$$

where $G(t, s) \in C([0, \omega] \times[0, \omega])$ is the Green function of the linear periodic boundary problem (2.2), which satisfies the positivity: $G(t, s)>0$ for every $(t, s) \in[0, \omega] \times[0, \omega]$.

For the details, see [14, Theorem 2.1 and Corollary 2.3].

For $m \in \mathbb{N}$, we use $C_{\omega}^{m}(\mathbb{R})$ to denote the $m$ th-order continuous differentiable $\omega$-periodic functions space. Let $X=C_{\omega}(\mathbb{R})$ be the Banach space of all continuous $\omega$-periodic functions equipped the norm $\|u\|=\max _{0 \leq t \leq \omega}|u(t)|$.

Let

$$
K_{0}=\{u \in X \mid u(t) \geq 0, t \in \mathbb{R}\}
$$


be the cone of all nonnegative functions in $X$. Then $X$ is an ordered Banach space by the cone $K_{0} . K_{0}$ has a nonempty interior

$$
\operatorname{int}\left(K_{0}\right)=\{u \in X \mid u(t)>0, t \in \mathbb{R}\} .
$$

Let

$$
\underline{G}=\min _{0 \leq t, s \leq \omega} G(t, s), \quad \bar{G}=\max _{0 \leq t, s \leq \omega} G(t, s) ; \quad \sigma=\underline{G} / \bar{G} .
$$

Lemma 2.2. Assume that (H1) holds, then for every $h \in X,(2.1)$ has a unique w-periodic solution $u$. Let $T: h \mapsto u$, then $T: X \rightarrow X$ is a completely continuous linear operator, and when $h \in K_{0}$, Th has the positivity estimate

$$
T h(t) \geq \sigma\|T h\|, \quad \forall t \in \mathbb{R} .
$$

Proof. Let $h \in X$. By Lemma 2.1, the linear periodic boundary problem (2.2) has a unique solution $u \in C^{2}[0, \omega]$ given by (2.3). We extend $u$ to a $\omega$-periodic function, which is still denoted by $u$, then $u:=T h \in C_{\omega}^{2}(\mathbb{R})$ is a unique $\omega$-periodic solution of (2.1). By (2.3),

$$
\operatorname{Th}(t)=\int_{0}^{\omega} G(t, s) h(s) d s, \quad t \in[0, \omega]
$$

From this we see that $T$ maps every bounded set in $X$ to a bounded equicontinuous set of $X$. Hence, by the Ascoli-Arzelà theorem, $T: X \rightarrow X$ is completely continuous.

Let $h \in K_{0}$. For every $t \in[0, \omega]$, from (2.8) it follows that

$$
\operatorname{Th}(t)=\int_{0}^{\omega} G(t, s) h(s) d s \leq \bar{G} \int_{0}^{\omega} h(s) d s,
$$

and therefore,

$$
\|T h\| \leq \bar{G} \int_{0}^{\omega} h(s) d s
$$

Using (2.8) and this inequality, we have that

$$
\begin{aligned}
T h(t) & =\int_{0}^{\omega} G(t, s) h(s) d s \geq \underline{G} \int_{0}^{\omega} h(s) d s \\
& =(\underline{G} / \bar{G}) \cdot \bar{G} \int_{0}^{\omega} h(s) d s \\
& \geq \sigma\|T h\| .
\end{aligned}
$$

Hence, by the periodicity of $u,(2.7)$ holds for every $t \in \mathbb{R}$. 
From (2.7) we easily see that $T\left(K_{0}\right) \subset \operatorname{int}\left(K_{0}\right)$; namely, $T: X \rightarrow X$ is a strongly positive linear operator. By the well-known Krein-Rutman theorem, the spectral radius $r(T)>0$ is a simple eigenvalue of $T$, and $T$ has a corresponding positive eigenfunction $\phi \in K_{0}$; that is,

$$
T \phi=r(T) \phi
$$

Since $\phi$ can be replaced by $c \phi$, where $c>0$ is a constant, we can choose $\phi \in K_{0}$ such that

$$
\int_{0}^{\omega} \phi(t) d t=1
$$

Set $\lambda_{1}=1 / r(T)$, then $\phi=T\left(\lambda_{1} \phi\right)$. By Lemma 2.2 and the definition of $T, \phi \in C_{\omega}^{2}(\mathbb{R})$ satisfies the differential equation

$$
\phi^{\prime \prime}(t)+a(t) \phi(t)=\lambda_{1} \phi(t), \quad t \in \mathbb{R}
$$

Thus, $\lambda_{1}$ is the minimum positive real eigenvalue of the linear equation (2.1) under the $\omega$ periodic condition. Summarizing these facts, we have the following lemma.

Lemma 2.3. Assume that (H1) holds, then there exist $\phi \in K_{0} \cap C_{\omega}^{2}(\mathbb{R})$ such that (2.13) and (2.14) hold.

Let $f: \mathbb{R} \times[0, \infty)^{n+1} \rightarrow[0, \infty)$ satisfy the assumption (H2). For every $u \in X$, set

$$
F(u)(t):=f\left(t, u(t), u\left(t-\tau_{1}\right), \ldots, u\left(t-\tau_{n}\right)\right), \quad t \in \mathbb{R} .
$$

Then $F: K_{0} \rightarrow K_{0}$ is continuous. Define a mapping $A: K_{0} \rightarrow X$ by

$$
A=T \circ F \text {. }
$$

By the definition of operator $T$, the $\omega$-periodic solution of (1.1) is equivalent to the fixed point of $A$. Choose a subcone of $K_{0}$ by

$$
K=\left\{u \in K_{0} \mid u(t) \geq \sigma\|u\|_{C}, t \in \mathbb{R}\right\} .
$$

By the strong positivity (2.7) of $T$ and the definition of $A$, we easily obtain the following.

Lemma 2.4. Assume that (H1) holds, then $A\left(K_{0}\right) \subset K$ and $A: K \rightarrow K$ is completely continuous.

Hence, the positive $\omega$-periodic solution of (1.1) is equivalent to the nontrivial fixed point of $A$. We will find the nonzero fixed point of $A$ by using the fixed-point index theory in cones.

We recall some concepts and conclusions on the fixed-point index in $[15,16]$. Let $X$ be a Banach space and $K \subset X$ a closed convex cone in $X$. Assume that $\Omega$ is a bounded open subset of $X$ with boundary $\partial \Omega$, and $K \cap \Omega \neq \emptyset$. Let $A: K \cap \bar{\Omega} \rightarrow K$ be a completely continuous 
mapping. If $A u \neq u$ for any $u \in K \cap \partial \Omega$, then the fixed-point index $i(A, K \cap \Omega, K)$ has definition. One important fact is that if $i(A, K \cap \Omega, K) \neq 0$, then $A$ has a fixed point in $K \cap \Omega$. The following two lemmas in [16] are needed in our argument.

Lemma 2.5. Let $\Omega$ be a bounded open subset of $X$ with $\theta \in \Omega$, and $A: K \cap \bar{\Omega} \rightarrow K$ a completely continuous mapping. If $\mu A u \neq u$ for every $u \in K \cap \partial \Omega$ and $0<\mu \leq 1$, then $i(A, K \cap \Omega, K)=1$.

Lemma 2.6. Let $\Omega$ be a bounded open subset of $X$ and $A: K \cap \bar{\Omega} \rightarrow K$ a completely continuous mapping. If there exists an $e \in K \backslash\{\theta\}$ such that $u-A u \neq \mu$ for every $u \in K \cap \partial \Omega$ and $\mu \geq 0$, then $i(A, K \cap \Omega, K)=0$.

\section{Main Results}

We consider the existence of positive $\omega$-periodic solutions of (1.1). Assume that $f: \mathbb{R} \times$ $[0, \infty)^{n+1} \rightarrow[0, \infty)$ satisfy $(\mathrm{H} 2)$. To be convenient, we introduce the notations

$$
\begin{aligned}
& f_{0}=\liminf _{\bar{x} \rightarrow 0^{+}} \min _{t \in[0, \omega]} \frac{f\left(t, x_{0}, x_{1}, \ldots, x_{n}\right)}{\bar{x}}, \\
& f^{0}=\limsup _{\bar{x} \rightarrow 0^{+}} \max _{t \in[0, \omega]} \frac{f\left(t, x_{0}, x_{1}, \ldots, x_{n}\right)}{\underline{x}}, \\
& f_{\infty}=\liminf _{\underline{x} \rightarrow+\infty} \min _{t \in[0, \omega]} \frac{f\left(t, x_{0}, x_{1}, \ldots, x_{n}\right)}{\bar{x}}, \\
& f^{\infty}=\limsup _{\underline{x} \rightarrow+\infty} \max _{t \in[0, \omega]} \frac{f\left(t, x_{0}, x_{1}, \ldots, x_{n}\right)}{\underline{x}},
\end{aligned}
$$

where $\bar{x}=\max \left\{x_{0}, x_{1}, \ldots, x_{n}\right\}$ and $\underline{x}=\min \left\{x_{0}, x_{1}, \ldots, x_{n}\right\}$. Our main results are as follows.

Theorem 3.1. Suppose that (H1)-(H3) hold. If $f$ satisfies the condition

(H4) $f^{0}<\lambda_{1}<f_{\infty}$,

then (1.1) has at least one positive w-periodic solution.

Theorem 3.2. Suppose that (H1)-(H3) hold. If $f$ satisfies the condition

(H5) $f^{\infty}<\lambda_{1}<f_{0}$,

then (1.1) has at least one positive w-periodic solution.

In Theorem 3.1, the condition (H4) allows $f\left(t, x_{0}, x_{1}, \ldots, x_{n}\right)$ to be superlinear growth on $x_{0}, x_{1}, \ldots, x_{n}$. For example,

$$
f\left(t, x_{0}, x_{1}, \ldots, x_{n}\right)=b_{0}(t) x_{0}^{2}+b_{1}(t) x_{1}^{2}+\cdots+b_{n}(t) x_{n}^{2}
$$

satisfies (H4) with $f^{0}=0$ and $f_{\infty}=+\infty$, where $b_{0}, b_{1}, \ldots, b_{n} \in C_{\omega}(\mathbb{R})$ are positive $\omega$-periodic functions. 
In Theorem 3.2, the condition (H5) allows $f\left(t, x_{0}, x_{1}, \ldots, x_{n}\right)$ to be sublinear growth on $x_{0}, x_{1}, \ldots, x_{n}$. For example,

$$
f\left(t, x_{0}, x_{1}, \ldots, x_{n}\right)=c_{0}(t) \sqrt{\left|x_{0}\right|}+c_{1}(t) \sqrt{\left|x_{1}\right|}+\cdots+c_{n}(t) \sqrt{\left|x_{n}\right|}
$$

satisfies (H5) with $f_{0}=+\infty$ and $f^{\infty}=0$, where $c_{0}, c_{1}, \ldots, c_{n} \in C_{\omega}(\mathbb{R})$ are positive $\omega$-periodic solution.

Applying Theorems 3.1 and 3.2 to (1.2), we have the following.

Corollary 3.3. Suppose that (H1)-(H3) hold. If the parameter $\lambda$ satisfies one of the following conditions
(1) $\lambda_{1} / f_{\infty}<\lambda<\lambda_{1} / f^{0}$,
(2) $\lambda_{1} / f_{0}<\lambda<\lambda_{1} / f^{\infty}$,

then (1.2) has at least one positive w-periodic solution.

This result improves and extends [5, Theorem 1.3].

Proof of Theorem 3.1. Let $K \subset X$ be the cone defined by (2.17) and $A: K \rightarrow K$ the operator defined by (2.16). Then the positive $\omega$-periodic solution of (1.1) is equivalent to the nontrivial fixed point of $A$. Let $0<r<R<+\infty$ and set

$$
\Omega_{1}=\{u \in X \mid\|u\|<r\}, \quad \Omega_{2}=\{u \in X \mid\|u\|<R\} .
$$

We show that the operator $A$ has a fixed point in $K \cap\left(\Omega_{2} \backslash \bar{\Omega}_{1}\right)$ when $r$ is small enough and $R$ large enough.

Since $f^{0}<\lambda_{1}$, by the definition of $f^{0}$, there exist $\varepsilon \in\left(0, \lambda_{1}\right)$ and $\delta>0$, such that

$$
f\left(t, x_{0}, x_{1}, \ldots, x_{n}\right) \leq\left(\lambda_{1}-\varepsilon\right) \underline{x}, \quad t \in[0, \omega], \bar{x} \in(0, \delta),
$$

where $\bar{x}=\max \left\{x_{0}, x_{1}, \ldots, x_{n}\right\}$ and $\underline{x}=\min \left\{x_{0}, x_{1}, \ldots, x_{n}\right\}$. Choosing $r \in(0, \delta)$, we prove that $A$ satisfies the condition of Lemma 2.5 in $K \cap \partial \Omega_{1}$; namely, $\mu A u \neq u$ for every $u \in K \cap \partial \Omega_{1}$ and $0<\mu \leq 1$. In fact, if there exist $u_{0} \in K \cap \partial \Omega_{1}$ and $0<\mu_{0} \leq 1$ such that $\mu_{0} A u_{0}=u_{0}$ and since $u_{0}=T\left(\mu_{0} F\left(u_{0}\right)\right)$, by definition of $T$ and Lemma 2.2, $u_{0} \in C_{\omega}^{2}(\mathbb{R})$ satisfies the differential equation

$$
u_{0}^{\prime \prime}(t)+a(t) u_{0}(t)=\mu_{0} F\left(u_{0}\right)(t), \quad t \in \mathbb{R},
$$

where $F(u)$ is defined by (2.15). Since $u_{0} \in K \cap \partial \Omega_{1}$, by the definitions of $K$ and $\Omega_{1}$, we have

$$
0<\sigma\left\|u_{0}\right\| \leq u_{0}(\tau) \leq\left\|u_{0}\right\|=r<\delta, \quad \forall \tau \in \mathbb{R} .
$$

This implies that

$$
0<\max \left\{u_{0}(t), u_{0}\left(t-\tau_{1}(t)\right), \ldots, u_{0}\left(t-\tau_{n}(t)\right)\right\}<\delta, \quad t \in \mathbb{R}
$$


From this and (3.5), it follows that

$$
\begin{aligned}
F\left(u_{0}\right)(t) & =f\left(t, u_{0}(t), u_{0}\left(t-\tau_{1}(t)\right), \ldots, u_{0}\left(t-\tau_{n}(t)\right)\right) \\
& \leq\left(\lambda_{1}-\varepsilon\right) \cdot \min \left\{u_{0}(t), u_{0}\left(t-\tau_{1}(t)\right), \ldots, u_{0}\left(t-\tau_{n}(t)\right)\right\} \\
& \leq\left(\lambda_{1}-\varepsilon\right) u_{0}(t), \quad t \in \mathbb{R} .
\end{aligned}
$$

By this inequality and (3.6), we have

$$
u_{0}^{\prime \prime}(t)+a(t) u_{0}(t)=\mu_{0} F\left(u_{0}\right)(t) \leq\left(\lambda_{1}-\varepsilon\right) u_{0}(t), \quad t \in \mathbb{R}
$$

Let $\phi \in K \cap C_{\omega}^{2}(\mathbb{R})$ be the function given in Lemma 2.4. Multiplying the inequality (3.10) by $\phi(t)$ and integrating on $[0, \omega]$, we have

$$
\int_{0}^{\omega}\left[u_{0}^{\prime \prime}(t)+a(t) u_{0}(t)\right] \phi(t) d t \leq\left(\lambda_{1}-\varepsilon\right) \int_{0}^{\omega} u_{0}(t) \phi(t) d t .
$$

For the left side of the above inequality using integration by parts, then using the periodicity of $u_{0}$ and $\phi$ and (2.14), we have

$$
\begin{aligned}
\int_{0}^{\omega}\left[u_{0}^{\prime \prime}(t)+a(t) u_{0}(t)\right] \phi(t) d t & =\int_{0}^{\omega} u_{0}(t)\left[\phi^{\prime \prime}(t)+a(t) \phi(t)\right] d t \\
& =\lambda_{1} \int_{0}^{\omega} u_{0}(x) \phi(t) d t
\end{aligned}
$$

Consequently, we obtain that

$$
\lambda_{1} \int_{0}^{\omega} u_{0}(x) \phi(t) d t \leq\left(\lambda_{1}-\varepsilon\right) \int_{0}^{\omega} u_{0}(x) \phi(t) d t
$$

Since $u_{0} \in K \cap \partial \Omega_{1}$, by the definition of $K$ and (2.13),

$$
\int_{0}^{\omega} u_{0}(x) \phi(t) d t \geq \sigma\left\|u_{0}\right\| \int_{0}^{\omega} \phi(t) d t=\sigma\left\|u_{0}\right\|>0 .
$$

From this and (3.13), we conclude that $\lambda_{1} \leq \lambda_{1}-\varepsilon$, which is a contradiction. Hence, $A$ satisfies the condition of Lemma 2.5 in $K \cap \partial \Omega_{1}$. By Lemma 2.5, we have

$$
i\left(A, K \cap \Omega_{1}, K\right)=1 .
$$

On the other hand, since $f_{\infty}>\lambda_{1}$, by the definition of $f_{\infty}$, there exist $\varepsilon_{1}>0$ and $H>0$ such that

$$
f\left(t, x_{0}, x_{1}, \ldots, x_{n}\right) \geq\left(\lambda_{1}+\varepsilon_{1}\right) \bar{x}, \quad t \in[0, \omega], \underline{x}>H,
$$


where $\bar{x}=\max \left\{x_{0}, x_{1}, \ldots, x_{n}\right\}$ and $\underline{x}=\min \left\{x_{0}, x_{1}, \ldots, x_{n}\right\}$. Choose $R>\max \{H / \sigma, \delta\}$ and $e(t) \equiv 1$. Clearly, $e \in K \backslash\{\theta\}$. We show that $A$ satisfies the condition of Lemma 2.6 in $K \cap \partial \Omega_{2}$; namely, $u-A u \neq \mu \phi$ for every $u \in K \cap \partial \Omega_{2}$ and $\mu \geq 0$. In fact, if there exist $u_{1} \in K \cap \partial \Omega_{2}$ and $\mu_{1} \geq 0$ such that $u_{1}-A u_{1}=\mu_{1} e$, since $u_{1}-\mu_{1} e=A u_{1}=T\left(F\left(u_{1}\right)\right)$, by the definition of $T$ and Lemma 2.2, $u_{1} \in C_{\omega}^{2}(\mathbb{R})$ satisfies the differential equation

$$
u_{1}^{\prime \prime}(t)+a(t)\left(u_{1}(t)-\mu_{1}\right)=F\left(u_{1}\right)(t), \quad t \in \mathbb{R} .
$$

Since $u_{1} \in K \cap \partial \Omega_{2}$, by the definitions of $K$ and $\Omega_{2}$, we have

$$
u_{1}(\tau) \geq \sigma\left\|u_{1}\right\|=\sigma R>H, \quad \forall \tau \in \mathbb{R}
$$

This means that

$$
\min \left\{u_{1}(t), u_{1}\left(t-\tau_{1}(t)\right), \ldots, u_{1}\left(t-\tau_{n}(t)\right)\right\}>H, \quad t \in \mathbb{R}
$$

Combining this with (3.16), we have that

$$
\begin{aligned}
F\left(u_{1}\right)(t) & =f\left(t, u_{1}(t), u_{1}\left(t-\tau_{1}(t)\right), \ldots, u_{1}\left(t-\tau_{n}(t)\right)\right) \\
& \geq\left(\lambda_{1}+\varepsilon_{1}\right) \cdot \max \left\{u_{1}(t), u_{1}\left(t-\tau_{1}(t)\right), \ldots, u_{1}\left(t-\tau_{n}(t)\right)\right\} \\
& \geq\left(\lambda_{1}+\varepsilon_{1}\right) u_{1}(t), \quad t \in \mathbb{R} .
\end{aligned}
$$

From this inequality and (3.17), it follows that

$$
u_{1}^{\prime \prime}(t)+a(t) u_{1}(t)=\mu_{1} a(t)+F\left(u_{1}\right)(t) \geq\left(\lambda_{1}+\varepsilon_{1}\right) u_{1}(t), \quad t \in \mathbb{R} .
$$

Multiplying this inequality by $\phi(t)$ and integrating on $[0, \omega]$, we have

$$
\int_{0}^{\omega}\left[u_{1}^{\prime \prime}(t)+a(t) u_{1}(t)\right] \phi(t) d t \geq\left(\lambda_{1}+\varepsilon_{1}\right) \int_{0}^{\omega} u_{1}(t) \phi(t) d t
$$

For the left side of the above inequality using integration by parts and (2.14), we have

$$
\begin{aligned}
\int_{0}^{\omega}\left[u_{1}^{\prime \prime}(t)+a(t) u_{1}(t)\right] \phi(t) d t & =\int_{0}^{\omega} u_{1}(t)\left[\phi^{\prime}(t)+a(t) \phi(t)\right] d t \\
& =\lambda_{1} \int_{0}^{\omega} u_{1}(x) \phi(t) d t
\end{aligned}
$$

From this and (3.22), it follows that

$$
\lambda_{1} \int_{0}^{\omega} u_{1}(x) \phi(t) d t \geq\left(\lambda_{1}+\varepsilon_{1}\right) \int_{0}^{\omega} u_{1}(x) \phi(t) d t .
$$


Since $u_{1} \in K \cap \partial \Omega_{2}$, by the definition of $K$ and (2.13), we have

$$
\int_{0}^{\omega} u_{1}(x) \phi(t) d t \geq \sigma\left\|u_{1}\right\| \int_{0}^{\omega} \phi(t) d t=\sigma\left\|u_{1}\right\|>0 .
$$

Hence, from (3.24) it follows that $\lambda_{1} \geq \lambda_{1}+\varepsilon_{1}$, which is a contradiction. Therefore, $A$ satisfies the condition of Lemma 2.6 in $K \cap \partial \Omega_{2}$. By Lemma 2.6, we have

$$
i\left(A, K \cap \Omega_{2}, K\right)=0 .
$$

Now by the additivity of the fixed-point index (3.15), and (3.26), we have

$$
i\left(A, K \cap\left(\Omega_{2} \backslash \bar{\Omega}_{1}\right), K\right)=i\left(A, K \cap \Omega_{2}, K\right)-i\left(A, K \cap \Omega_{1}, K\right)=-1 .
$$

Hence $A$ has a fixed point in $K \cap\left(\Omega_{2} \backslash \bar{\Omega}_{1}\right)$, which is a positive $\omega$-periodic solution of (1.1).

Proof of Theorem 3.2. Let $\Omega_{1}, \Omega_{2} \subset X$ be defined by (3.4). We prove that the operator $A$ defined by (2.16) has a fixed point in $K \cap\left(\Omega_{2} \backslash \bar{\Omega}_{1}\right)$ if $r$ is small enough and $R$ is large enough.

By $f_{0}>\lambda_{1}$ and the definition of $f_{0}$, there exist $\varepsilon>0$ and $\delta>0$, such that

$$
f\left(t, x_{0}, x_{1}, \ldots, x_{n}\right) \geq\left(\lambda_{1}+\varepsilon\right) \bar{x}, \quad t \in[0, \omega], \bar{x} \in(0, \delta),
$$

where $\bar{x}=\max \left\{x_{0}, x_{1}, \ldots, x_{n}\right\}$. Let $r \in(0, \delta)$ and $e(t) \equiv 1$. We prove that $A$ satisfies the condition of Lemma 2.6 in $K \cap \partial \Omega_{1}$; namely, $u-A u \neq \mu$ e for every $u \in K \cap \partial \Omega_{1}$ and $\mu \geq 0$. In fact, if there exist $u_{0} \in K \cap \partial \Omega_{1}$ and $\mu_{0} \geq 0$ such that $u_{0}-A u_{0}=\mu_{0} e$ and since $u_{0}-\mu_{0} e=A u_{0}=$ $T\left(F\left(u_{0}\right)\right)$, by the definition of $T$ and Lemma $2.2, u_{0} \in C_{\omega}^{2}(\mathbb{R})$ satisfies the differential equation

$$
u_{0}^{\prime \prime}(t)+a(t)\left(u_{0}(t)-\mu_{0}\right)=F\left(u_{0}\right)(t), \quad t \in \mathbb{R} .
$$

Since $u_{0} \in K \cap \partial \Omega_{1}$, by the definitions of $K$ and $\Omega_{1}$, $u_{0}$ satisfies (3.7), and hence (3.8) holds. From (3.8) and (3.28), it follows that

$$
\begin{aligned}
F\left(u_{0}\right)(t) & =f\left(t, u_{0}(t), u_{0}\left(0-\tau_{1}(t)\right), \ldots, u_{0}\left(t-\tau_{n}(t)\right)\right) \\
& \geq\left(\lambda_{1}+\varepsilon\right) \cdot \max \left\{u_{0}(t), u_{0}\left(t-\tau_{1}(t)\right), \ldots, u_{0}\left(t-\tau_{n}(t)\right)\right\} \\
& \geq\left(\lambda_{1}+\varepsilon\right) u_{0}(t), \quad t \in \mathbb{R} .
\end{aligned}
$$

By this and (3.29), we obtain that

$$
u_{0}^{\prime \prime}(t)+a(t) u_{0}(t)=\mu_{0} a(t)+F\left(u_{0}\right)(t) \geq\left(\lambda_{1}+\varepsilon\right) u_{0}(t), \quad t \in \mathbb{R} .
$$

Multiplying this inequality by $\phi(t)$ and integrating on $[0, \omega]$, we have

$$
\int_{0}^{\omega}\left[u_{0}^{\prime \prime}(t)+a(t) u_{0}(t)\right] \phi(t) d t \geq\left(\lambda_{1}+\varepsilon\right) \int_{0}^{\omega} u_{0}(t) \phi(t) d t .
$$


For the left side of this inequality using integration by parts and (2.14), we have

$$
\begin{aligned}
\int_{0}^{\omega}\left[u_{0}^{\prime \prime}(t)+a(t) u_{0}(t)\right] \phi(t) d t & =\int_{0}^{\omega} u_{0}(t)\left[\phi^{\prime}(t)+a(t) \phi(t)\right] d t \\
& =\lambda_{1} \int_{0}^{\omega} u_{0}(x) \phi(t) d t
\end{aligned}
$$

From this and (3.32), it follows that

$$
\lambda_{1} \int_{0}^{\omega} u_{1}(x) \phi(t) d t \geq\left(\lambda_{1}+\varepsilon_{1}\right) \int_{0}^{\omega} u_{1}(x) \phi(t) d t
$$

Since $u_{0} \in K \cap \partial \Omega_{1}$, from the definition of $K$ and (2.13) it follows that (3.14) holds. By (3.14) and (3.34), we see that $\lambda_{1} \geq \lambda_{1}+\varepsilon$, which is a contradiction. Hence, $A$ satisfies the condition of Lemma 2.6 in $K \cap \partial \Omega_{1}$. By Lemma 2.6, we have

$$
i\left(A, K \cap \Omega_{1}, K\right)=0 .
$$

Since $f^{\infty}<\lambda_{1}$, by the definition of $f^{\infty}$, there exist $\varepsilon_{1} \in\left(0, \lambda_{1}\right)$ and $H>0$ such that

$$
f\left(t, x_{0}, x_{1}, \ldots, x_{n}\right) \leq\left(\lambda_{1}-\varepsilon_{1}\right) \underline{x}, \quad t \in[0, \omega], \underline{x}>H,
$$

where $\underline{x}=\min \left\{x_{0}, x_{1}, \ldots, x_{n}\right\}$. Choosing $R>\max \{H / \sigma, \delta\}$, we show that $A$ satisfies the condition of Lemma 2.5 in $K \cap \partial \Omega_{2}$; namely, $\mu A u \neq u$ for every $u \in K \cap \partial \Omega_{2}$ and $0<\mu \leq 1$. In fact, if there exist $u_{1} \in K \cap \partial \Omega_{2}$ and $0<\mu_{1} \leq 1$ such that $\mu_{1} A u_{1}=u_{1}$, since $u_{1}=T\left(\mu_{1} F\left(u_{1}\right)\right)$, by the definition of $T$ and Lemma 2.2, $u_{1} \in C_{\omega}^{2}(\Omega)$ satisfies the differential equation

$$
u_{1}^{\prime \prime}(t)+a(t) u_{1}(t)=\mu_{1} F\left(u_{1}\right)(t), \quad t \in \mathbb{R} .
$$

Since $u_{1} \in K \cap \partial \Omega_{2}$, by the definitions of $K$ and $\Omega_{2}, u_{1}$ satisfies (3.18), and hence (3.19) holds. By (3.19) and (3.36), we have

$$
\begin{aligned}
F\left(u_{1}\right)(t) & =f\left(t, u_{1}(t), u_{1}\left(t-\tau_{1}(t)\right), \ldots, u_{1}\left(t-\tau_{n}(t)\right)\right) \\
& \leq\left(\lambda_{1}-\varepsilon_{1}\right) \cdot \min \left\{u_{1}(t), u_{1}\left(t-\tau_{1}(t)\right), \ldots, u_{1}\left(t-\tau_{n}(t)\right)\right\} \\
& \leq\left(\lambda_{1}-\varepsilon_{1}\right) u_{1}(t), \quad t \in \mathbb{R} .
\end{aligned}
$$

From this inequality and (3.37), it follows that

$$
u_{1}^{\prime \prime}(t)+a(t) u_{1}(t)=\mu_{1} F\left(u_{1}\right)(t) \leq\left(\lambda_{1}-\varepsilon_{1}\right) u_{1}(t), \quad t \in \mathbb{R} .
$$

Multiplying this inequality by $\phi(t)$ and integrating on $[0, \omega]$, we have

$$
\int_{0}^{\omega}\left[u_{1}^{\prime \prime}(t)+a(t) u_{1}(t)\right] \phi(t) d t \leq\left(\lambda_{1}-\varepsilon_{1}\right) \int_{0}^{\omega} u_{1}(t) \phi(t) d t
$$


For the left side of this inequality using integration by parts and (2.14), we have

$$
\begin{aligned}
\int_{0}^{\omega}\left[u_{1}^{\prime \prime}(t)+a(t) u_{1}(t)\right] \phi(t) d t & =\int_{0}^{\omega} u_{1}(t)\left[\phi^{\prime \prime}(t)+a(t) \phi(t)\right] d t \\
& =\lambda_{1} \int_{0}^{\omega} u_{1}(x) \phi(t) d t
\end{aligned}
$$

Consequently, we obtain that

$$
\lambda_{1} \int_{0}^{\omega} u_{1}(x) \phi(t) d t \leq\left(\lambda_{1}-\varepsilon_{1}\right) \int_{0}^{\omega} u_{1}(x) \phi(t) d t
$$

Since $u_{1} \in K \cap \partial \Omega_{2}$, by the definition of $K$ and (2.13) we see that (3.25) holds. From (3.25) and (3.42), we see that $\lambda_{1} \leq \lambda_{1}-\varepsilon_{1}$, which is a contradiction. Hence, $A$ satisfies the condition of Lemma 2.5 in $K \cap \partial \Omega_{2}$. By Lemma 2.5 we have

$$
i\left(A, K \cap \Omega_{2}, K\right)=1 .
$$

Now, from (3.35) and (3.43) it follows that

$$
i\left(A, K \cap\left(\Omega_{2} \backslash \bar{\Omega}_{1}\right), K\right)=i\left(A, K \cap \Omega_{2}, K\right)-i\left(A, K \cap \Omega_{1}, K\right)=1 .
$$

Hence, $A$ has a fixed point in $K \cap\left(\Omega_{2} \backslash \bar{\Omega}_{1}\right)$, which is a positive $\omega$-periodic solution of (1.1).

\section{Acknowledgment}

This research supported by NNSF of China (11261053 and 11061031).

\section{References}

[1] B. Liu, "Periodic solutions of a nonlinear second-order differential equation with deviating argument," Journal of Mathematical Analysis and Applications, vol. 309, no. 1, pp. 313-321, 2005.

[2] J.-W. Li and S. S. Cheng, "Periodic solutions of a second order forced sublinear differential equation with delay," Applied Mathematics Letters, vol. 18, no. 12, pp. 1373-1380, 2005.

[3] Y. Wang, H. Lian, and W. Ge, "Periodic solutions for a second order nonlinear functional differential equation," Applied Mathematics Letters, vol. 20, no. 1, pp. 110-115, 2007.

[4] J. Wu and Z. Wang, "Two periodic solutions of second-order neutral functional differential equations," Journal of Mathematical Analysis and Applications, vol. 329, no. 1, pp. 677-689, 2007.

[5] Y. Wu, "Existence nonexistence and multiplicity of periodic solutions for a kind of functional differential equation with parameter," Nonlinear Analysis. Theory, Methods E Applications, vol. 70, no. 1, pp. 433-443, 2009.

[6] C. Guo and Z. Guo, "Existence of multiple periodic solutions for a class of second-order delay differential equations," Nonlinear Analysis. Real World Applications, vol. 10, no. 5, pp. 3285-3297, 2009.

[7] B. Yang, R. Ma, and C. Gao, "Positive periodic solutions of delayed differential equations," Applied Mathematics and Computation, vol. 218, no. 8, pp. 4538-4545, 2011.

[8] Y. Li, "Positive periodic solutions of second-order differential equations with delays," Abstract and Applied Analysis, vol. 2012, Article ID 829783, 13 pages, 2012. 
[9] Y. X. Li, "Positive periodic solutions of nonlinear second order ordinary differential equations," Acta Mathematica Sinica, vol. 45, no. 3, pp. 481-488, 2002.

[10] Y. Li, "Positive periodic solutions of first and second order ordinary differential equations," Chinese Annals of Mathematics B, vol. 25, no. 3, pp. 413-420, 2004.

[11] Y. Li and H. Fan, "Existence of positive periodic solutions for higher-order ordinary differential equations," Computers \& Mathematics with Applications, vol. 62, no. 4, pp. 1715-1722, 2011.

[12] F. Li and Z. Liang, "Existence of positive periodic solutions to nonlinear second order differential equations," Applied Mathematics Letters, vol. 18, no. 11, pp. 1256-1264, 2005.

[13] B. Liu, L. Liu, and Y. Wu, "Existence of nontrivial periodic solutions for a nonlinear second order periodic boundary value problem," Nonlinear Analysis. Theory, Methods E Applications, vol. 72, no. 7-8, pp. 3337-3345, 2010.

[14] P. J. Torres, "Existence of one-signed periodic solutions of some second-order differential equations via a Krasnoselskii fixed point theorem," Journal of Differential Equations, vol. 190, no. 2, pp. 643-662, 2003.

[15] K. Deimling, Nonlinear Functional Analysis, Springer-Verlag, New York, NY, USA, 1985.

[16] D. J. Guo and V. Lakshmikantham, Nonlinear Problems in Abstract Cones, vol. 5, Academic Press, New York, NY, USA, 1988. 


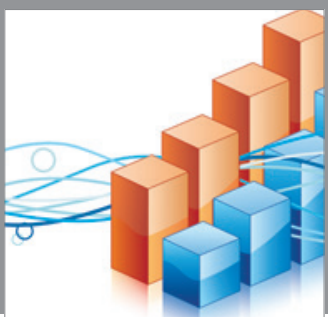

Advances in

Operations Research

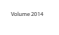

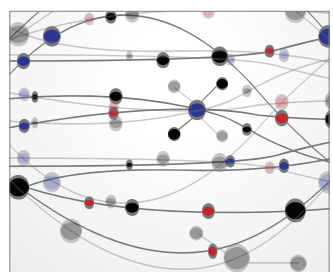

\section{The Scientific} World Journal
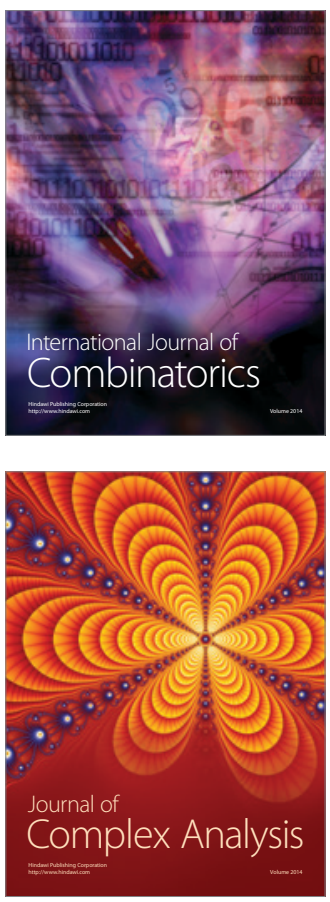

International Journal of

Mathematics and

Mathematical

Sciences
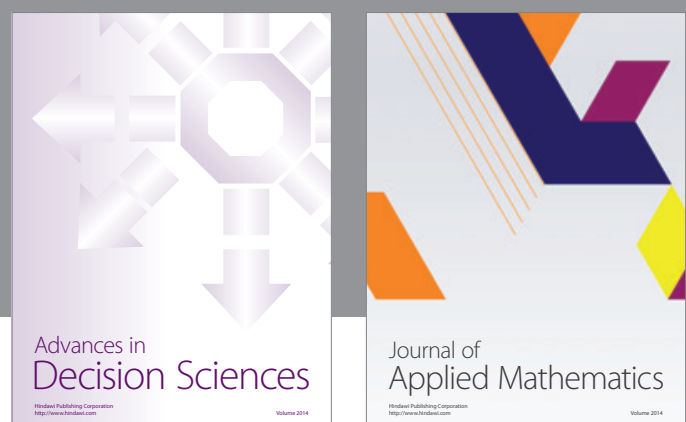

Journal of

Applied Mathematics
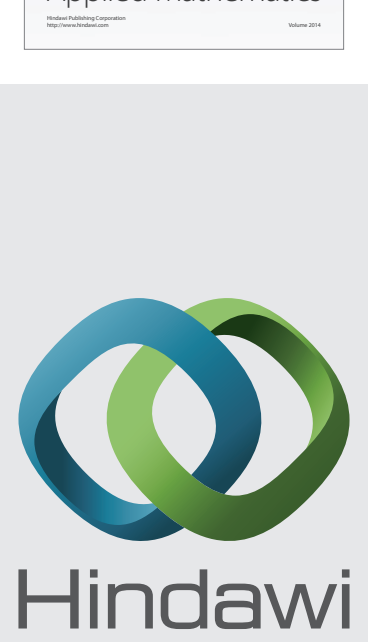

Submit your manuscripts at http://www.hindawi.com
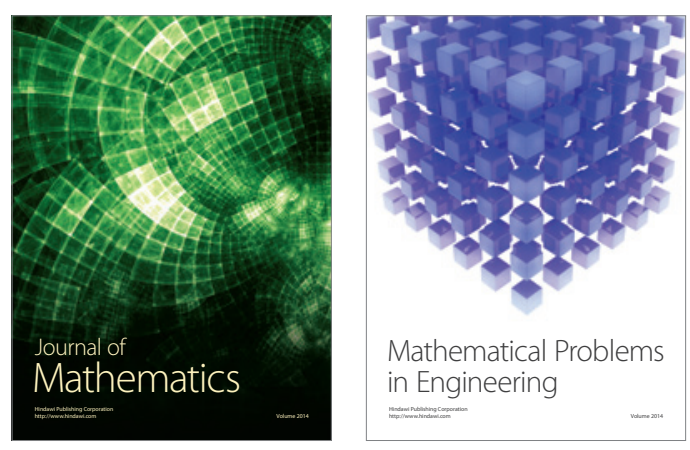

Mathematical Problems in Engineering
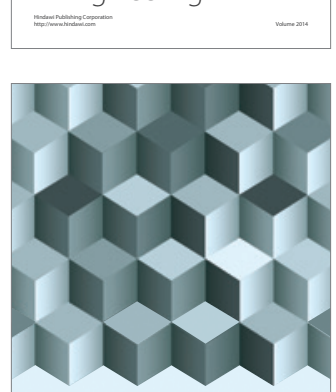

Journal of

Function Spaces
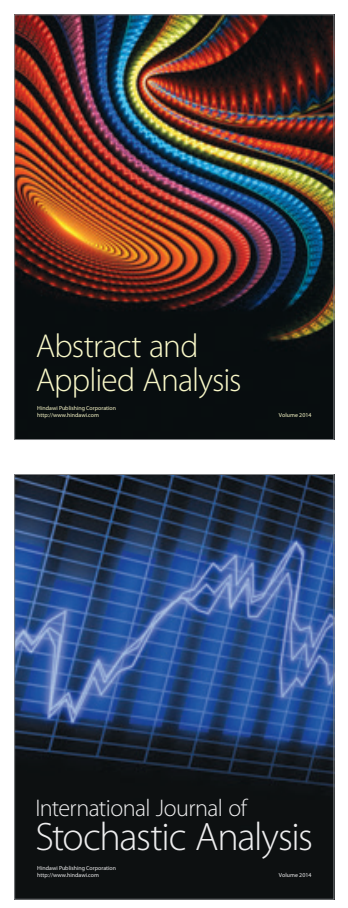

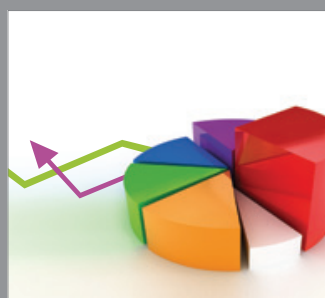

ournal of

Probability and Statistics

Promensencen
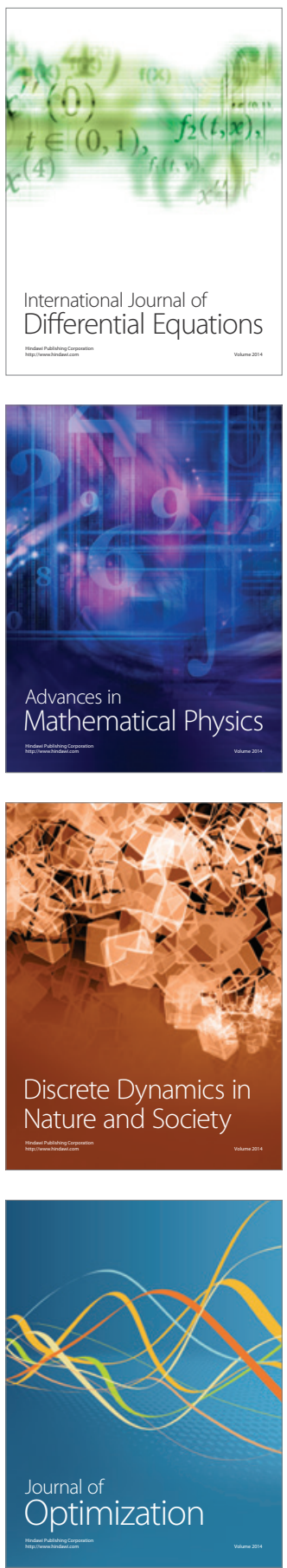